\title{
Structural Characterization and Statistical Properties of Two-Dimensional Granular Systems During the Compaction
}

\author{
S. Živković ${ }^{a}$, Z.M. JAKŠIĆc ${ }^{a}$, D. Arsenović ${ }^{a}$, LJ. Budinski-Petković ${ }^{b}$, S.B. VRhovaC ${ }^{a}$ \\ ${ }^{a}$ Institute of Physics, University of Belgrade, P.O. Box 68, Zemun 11080, Belgrade, Serbia \\ ${ }^{b}$ Faculty of Engineering, Trg. D. Obradovića 6, Novi Sad 21000, Serbia
}

\begin{abstract}
We study the compaction dynamics of frictional hard disks in two dimensions, subjected to vertical shaking, by numerical simulation. Shaking is modeled by a series of vertical expansions of the disk packing, followed by dynamical recompression of the assembly under the action of gravity. The second phase of the shake cycle is based on an efficient event-driven molecular-dynamics algorithm. We analyze the compaction dynamics for various values of the friction coefficient and the coefficient of normal restitution. The granular organization at local level was studied by analyzing the shape factor $\xi$ of the local volumes, associated with a natural way of subdividing the volume into local parts - the Voronoi partition. It gives a clear physical picture of the competition between less and more ordered domains of particles during the compaction. We calculate the distribution of the shape-factor for packings at different stages of the compaction process. We have also investigated a two-dimensional granular medium experimentally. We prepared the granular packings of metallic cylinders of diameters 4, 5, and $6 \mathrm{~mm}$. The distributions of the shape-factor obtained numerically for various tapping intensities are consistent with our experimental results.
\end{abstract}

PACS: 45.70.-n, 45.70.Cc, 81.05.Rm, 61.43.Bn

\section{Introduction}

The vibratory compaction of granular materials has long been of importance in many technological applications. The underlying dynamic and structural properties of the compaction process are a subject of great interest for physicists in recent years [1-5]. The phenomenon of granular compaction involves the increase of the density of granular material subjected to shaking, tapping or, more generally, to some kind of external excitation.

First, we study the compaction dynamics of frictional hard disks in two dimensions, subjected to vertical shaking, by numerical simulation. During the redeposition of the packing, the disks undergo instantaneous, inelastic binary collisions and propagate under gravity in between the collision events. Their settlement is terminated when the total kinetic energy of each disk falls below some threshold value. The algorithm employed in the present paper describes relatively accurately the quite complex succession of collisions in a shaken packing and provides a realistic information about its microstructural transformations during the compaction.

Our numerical simulation is based on the ideas of Barker and Mehta [6, 7] and Bideau and coworkers [8], with modifications aiming at more realistic treatment of gravitational redeposition of granular particles. The previous models [6-8] may have limited applications because of not taking into account the friction and other interparticle forces.

In addition, we investigate experimentally a twodimensional (2D) dense, disordered granular medium composed of rigid noncohesive grains. Our experimental apparatus is adapted from a geometry introduced by
Kolb et al. [9]. We study the dependence of the microstructural properties of packings on some parameters like the packing fraction of granular medium and compare the simulation results to the experimental ones. We apply the novel concept of shape factor, recently introduced by Moucka and Nezbeda [10], to measure in detail the topology of the Voronoi cells during the penetration process. The shape factor is a dimensionless measure of deviation of the Voronoi cells from circularity. This quantity was recently used to study the crystallization of two-dimensional systems, both in simulation [10] and experiment [11]. Since the shape factor clearly indicates the presence of domains made up of different Voronoi polygons, it gives a relevant physical picture of the competition between less and more ordered domains in the packing.

The plan of the paper is as follows. The simulation methodology is presented in Sec. 2, and the numerical results are presented in Sec. 3. The experimental results are reported and discussed in Sec. 4. Finally, Sec. 5 contains concluding remarks and comments.

\section{Simulation method}

The compaction of $N=1000$ monosize disks of diameter $d$ and mass $m$ under consecutive taps is studied in a rectangular container of width $L=1$, with a flat bottom at $y=0$ and opened top. A gravitational field $\boldsymbol{g}$ acts along the negative $y$ direction. One shake cycle of the granular assembly (corresponding to one time step of our simulation) is decomposed in two stages: 1) vertical dilatation of the disk packing, in proportion to the 
shaking acceleration $\Gamma$ and 2) formation of static granular pack in the presence of gravity. Repeated application of the shaking algorithm builds a sequence of static packings where each new packing is built from its predecessor. Shaking acceleration $\Gamma$ is defined as the ratio of the peak acceleration of a tap to the gravitational acceleration.

In the first phase of the shake cycle, free volume is introduced uniformly throughout the whole packing. A disk at height $y$ is raised to a new height $y^{\prime}=(1+\xi) y$. This models the dilation phase of a vibrated granular medium. In that sense, the parameter $\xi$ in our model plays a similar role as $\Gamma$ in real experiments. After dilation, we give a random initial velocity to each disk, in such a way that the total momentum is equal to zero. The choice of the initial velocity distribution function does not play a substantial role in our simulation.

In the second phase of the shake cycle, the packing is compressed under gravity, using an efficient event-driven molecular-dynamics algorithm [12]. The disks are assumed to be inelastic with rough s subject to Coulomb friction. In the event driven method the disks follow an undisturbed motion, under the influence of gravity, until an event occurs. An event is either the collision of two disks or the collision of one disk with the wall. The trajectories between collision events are parabolic arcs due to the presence of the gravitational field. Particle collisions are modeled using the Walton model $[13,14]$. This model implies that two disks at contact either slide, following the Coulomb's law, or stick together. The collision rule (given in detail in the [13]) takes into account a reduction of normal relative velocity of the two particles and a reduction of total tangential relative velocity. The collisions with the wall are treated in the same way as the collisions between particles, except the wall has an infinite mass. To prevent an inelastic collapse [15, 16], we use a coefficient of restitution which depends on the relative colliding velocity of the particles [17].

Due to dissipative collisions, the potential energy of the system tends towards a constant, while its kinetic energy tends towards zero in the long time limit. A static configuration of disks with zero kinetic energy is reached by imposing the following stopping criteria. A disk is considered to be at rest if both translational and rotational kinetic energy of the disk in the last ten collisions falls below a threshold value $E_{\mathrm{tr}}^{(t)}$ and $E_{\mathrm{tr}}^{(r)}$, respectively. Here $E_{\mathrm{tr}}^{(t)}$ and $E_{\mathrm{tr}}^{(r)}$ are the free parameters that are chosen to optimize the computational method. In this study, we used very small values $E_{\mathrm{tr}}^{(t)}=5 \times 10^{-5}$ and $E_{\mathrm{tr}}^{(r)}=10^{-6}$, in dimensionless units in which the container width $L$, mass $m$ and gravitational acceleration $g$ all equal unity. However, in a subsequent presentation of the results on the compaction dynamics, the time $T$ is measured in the number of taps.

These simulations were performed on the parallel cluster computer consisting of 100 Intel processors. A typical simulation of entire compaction process used $3 \mathrm{CPU}$ days on a single processor.

\section{Results and numerical simulations}

In the simulations, we employ the Walton model $[13,14]$ that captures the major features of granular interactions. We describe the roughness of the surfaces and the energy dissipation, using the parameters $\mu$ (coefficient of friction) and $\beta_{0}$ (coefficient of maximum tangential restitution). Further mechanisms of energy dissipation are the permanent deformation of a particle during the contact and the transfer of kinetic energy to thermal energy. We account for these effects using the velocity dependent coefficient of normal restitution $\varepsilon$. In order to examine the effects of inelastic and friction properties of grains on compaction dynamics, we used two sets of material parameters. More dissipative and rough disks (referred hereafter as disks (A)) are characterized by coefficients $\varepsilon_{0}=0.6$ and $\mu=0.4$, whereas with parameters $\varepsilon_{0}=0.9$ and $\mu=0.2$ we characterize the less dissipative disks (disks (B)). For all of the simulations in this paper we set $\beta_{0}=0.5$ [18]. We used the same inelasticity and friction coefficients for the grain-grain and the grain-wall collisions including the horizontal base.

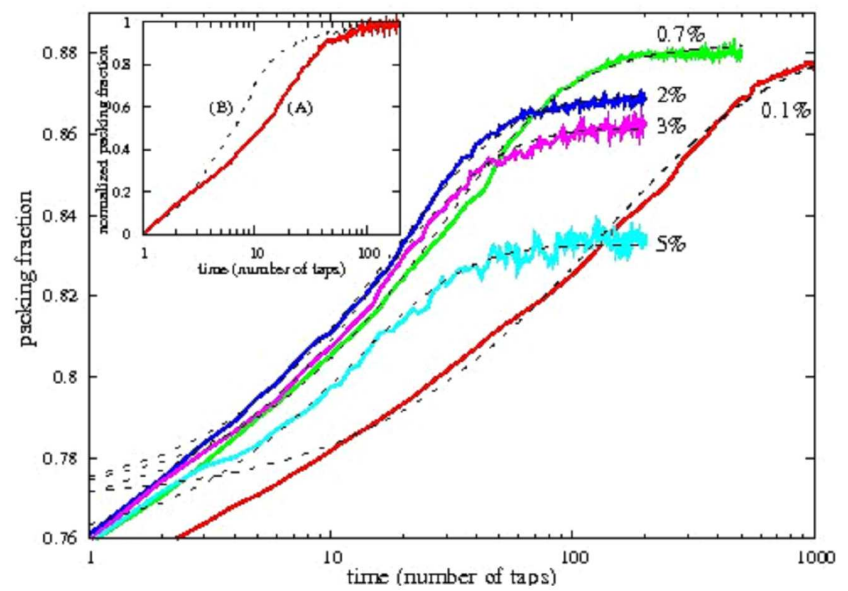

Fig. 1. Temporal evolution of the packing fraction $\rho(t)$ obtained for the grains of type (A) and for various tapping intensities $\xi=0.1 \%, 0.7 \%, 2 \%, 3 \%$ and $5 \%$. The dashed curves are the Mittag-Leffler fits of Eq. (1). Inset: evolution of the normalized packing fraction for two kinds of the grains (disks (A) - solid line and disks (B) - dashed line), at $\xi=3 \%$.

The variation of the packing fraction $\rho(t)$ with the number of shakes $t$ for several tapping intensities $\xi$ is presented in Fig. 1, where more dissipative grains (disks (A)) have been used. The inset of Fig. 1 compares the evolution of the normalized packing fraction $\tilde{\rho}(t)=$ $(\rho(t)-\rho(0)) /(\rho(1)-\rho(0))$ for the two kinds of grains (A) and (B), for $\xi=3 \%$. The simulation curves are in a good qualitative agreement with the experimental data obtained in the experiments with a reduced lateral confinement $[2,3]$. We have observed that the compaction dynamics gets slower when the tapping inten- 
sity $\xi$ decreases. Actually, when a small tapping intensity is applied, the evolution of the density toward the steady-state value $\rho_{\infty}$ takes place on a much wider time scale and finally a larger value of the asymptotic packing fraction is achieved. Several empirical and theoretical models have been proposed in the literature to describe the density relaxation, primarily in tapping experiments [5, 19-21]. Recently, we showed [22] that the resulting compaction dynamics is well described by the fractional Mittag-Leffler law for relaxation:

$$
\begin{aligned}
& \rho(t)=\rho_{\infty}-\Delta \rho E_{\alpha}\left[-(t / \tau)^{\alpha}\right], \\
& \Delta \rho \rho_{\infty}-\rho_{0}, \quad 0<\alpha<1,
\end{aligned}
$$

where $\rho_{0}$ is the initial packing fraction and $\rho \infty$ is the packing fraction at the steady state. $E_{\alpha}$ denotes the Mittag-Leffler function of order $\alpha$ [23]. It is interesting to note that the Mittag-Leffler model has been shown to describe the relaxation of granular materials under very different modes of external excitations: that is tapping [22], vibration [24], and thermal cycling [25].

A further insight into the dynamical mechanisms which take place during the compaction can be gained by analyzing the microstructural properties of the packings.

The study of how space is shared among the grains is essential for understanding how efficiently the disks are arranged locally. The first problem to address is how to divide the whole space occupied by the granular material into parts associated with the local environment around each disk. A natural way to subdivide the space into smaller portions is the Voronoi Tesselation (VT) [26]. For a given two-dimensional distribution of disks the VT is a uniquely defined set of convex cells, each of which encloses one and only one of these disks. A Voronoi cell (polygon in 2D) associated with a disk is defined as an assembly of points which are closer to that disk than to any of the other disks in the packing. Two disks sharing a common cell edge are neighbors. Each vertex of this tessellation is equidistant to three neighboring disks. The individual characteristics of Voronoi cells as, e.g., number of edges, circumference, surface, etc., are not able to characterize the tiny structural effects $[27,28]$ in detail, and have very poor sensitivity to the packing properties [29]. However, a better indicator of structural changes is the shape factor $\zeta$ (parameter of nonsphericity) which combines the circumference $C$ and the area $S$ of Voronoi cells [10, 30], which is defined by

$$
\zeta=\frac{C^{2}}{4 \pi S}
$$

For a circle, this coefficient is equal to 1. For a convex polygon, the more anisotropic the polygon, the higher is $\zeta>1$.

The shape factor is able to identify the occurrence of different domains in numerically obtained packings of particles. Every domain is made up of the grains whose Voronoi polygons have similar values of the shape factor. In order to quantify the structural changes in the packings during the compaction in a better way, we consider here the probability distribution $P(\zeta)$ of the shape fac- tor $\zeta$. The distribution function $P(\zeta)$ is related to the probability of finding the Voronoi cell with a shape factor $\zeta$. It is normalized to unity, namely, $\int_{0}^{\infty} \mathrm{d} \zeta P(\zeta)=1$. Note that the distribution of the shape factor (the occurrence probability of different polygons) for the fluid of two-dimensional hard disks depends on the packing fraction [10].

Figures 2 and 3 show the temporal evolution of the $P(\zeta)$ for the more dissipative disks (A) at two different tapping intensities, $\xi=0.7 \%, 3 \%$. In the initial stage of density relaxation the particles are distributed randomly, no specific figures are formed, and we thus get a flat and very broad distribution $P(\zeta)$. The distribution $P(\zeta)$ tends to narrow during the compaction. As the density increases, the distribution becomes more localized around the lowest values of the shape factor (for a regular hexagon, $\left.\zeta=6 / \sqrt{3 \pi^{2}} \approx 1.103\right)$. The curves of distribution $P(\zeta)$ are asymmetric with a long tail on the right-hand side, which progressively reduces while the packing structure gets more compact. This narrowing of the probability distribution $P(\zeta)$ corresponds to the increase of the fraction of more regular (round) figures. In other words, the Voronoi cells become more circular at higher values of the packing fraction and their occurrence starts prevailing. As one can see from Figs. 2 and 3, the narrowing of the $P(\zeta)$ during the compaction is more pronounced for the larger tapping intensity $\xi$.

The probability distribution $P(\zeta)$ is also sensitive to the material properties of disks.

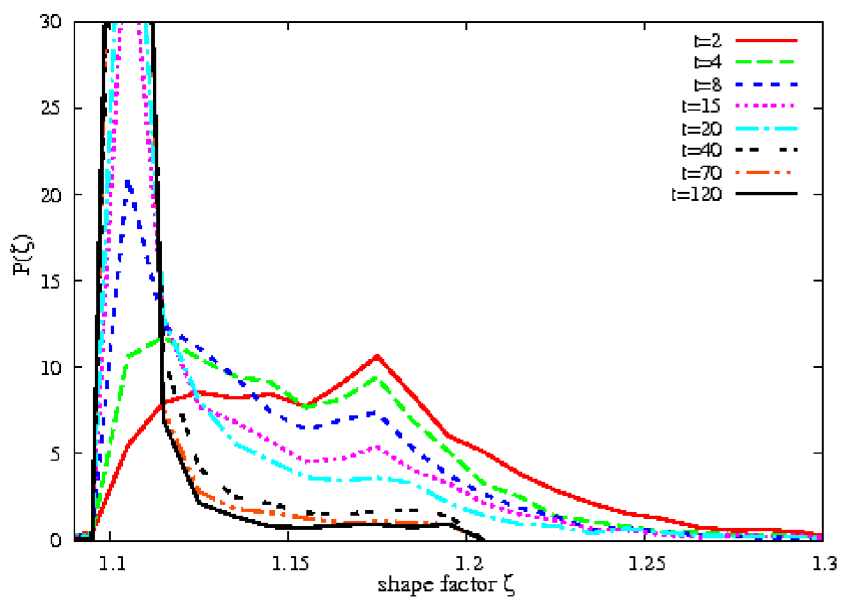

Fig. 2. The temporal evolution of the probability distribution $P(\zeta)$ of the shape factor $\zeta$ for the more dissipative disks (A) at tapping intensity $\xi=3 \%$.

Figures 4 and 5 show the temporal evolution of the $P(\zeta)$ for less dissipative disks (B) at two different tapping intensities, $\xi=0.7 \%, 3 \%$.

Here again, the curves of distribution $P(\zeta)$ are asymmetric with a quite long tail on the right-hand side. The tails of such distributions reduces more quickly for the less dissipative grains (B) than for the more dissipative grains (A). Indeed, for the less dissipative disks (B) there 


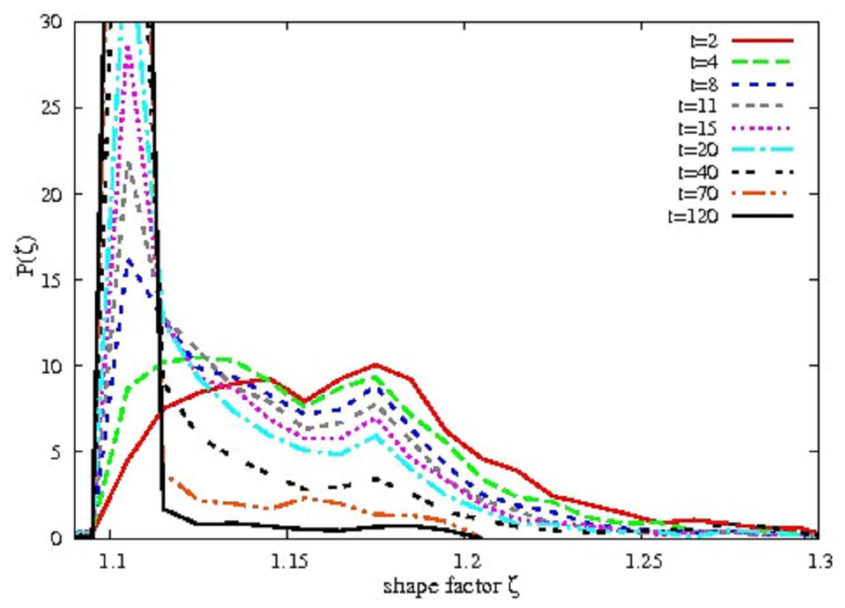

Fig. 3. The temporal evolution of the probability distribution $P(\zeta)$ of the shape factor $\zeta$ for the more dissipative disks (A) at tapping intensity $\xi=0.7 \%$.

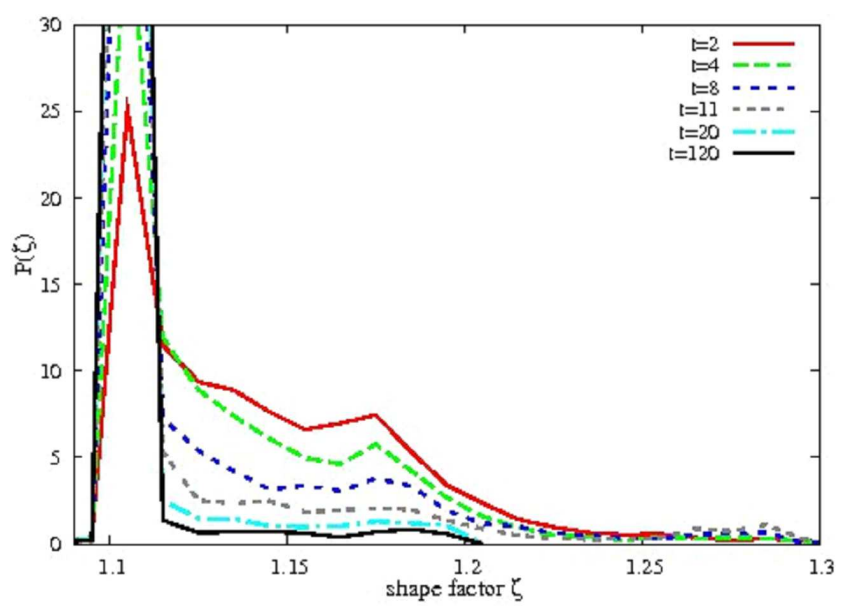

Fig. 4. The temporal evolution of the probability distribution $P(\zeta)$ of the shape factor $\zeta$ for the less dissipative disks (B) at tapping intensity $\xi=3 \%$.

is a rapid approach to the steady-state density. However, for a given tapping intensity $\xi$, the system of disks (B) achieves a larger value of the asymptotic density than the system of more dissipative disks (A). Consequently, in the case of disks (B) the system ends up in steadystate configurations where large clusters of nearregular Voronoi cells (hexagonal domains) are found.

\section{Experimental procedure and results}

Experiments were carried out on a 2D granular medium, i.e., the motion of the grains was confined to a plane. The host granular packing is constituted of metallic cylinders contained in a rectangular box made of two parallel glass plates, with an inner gap of thickness $3.4 \mathrm{~mm}$, slightly larger than the height of the cylinders, $h=3.00 \pm 0.01 \mathrm{~mm}$. The axes of the cylinders are perpendicular to the low frictional glass plates. The lateral

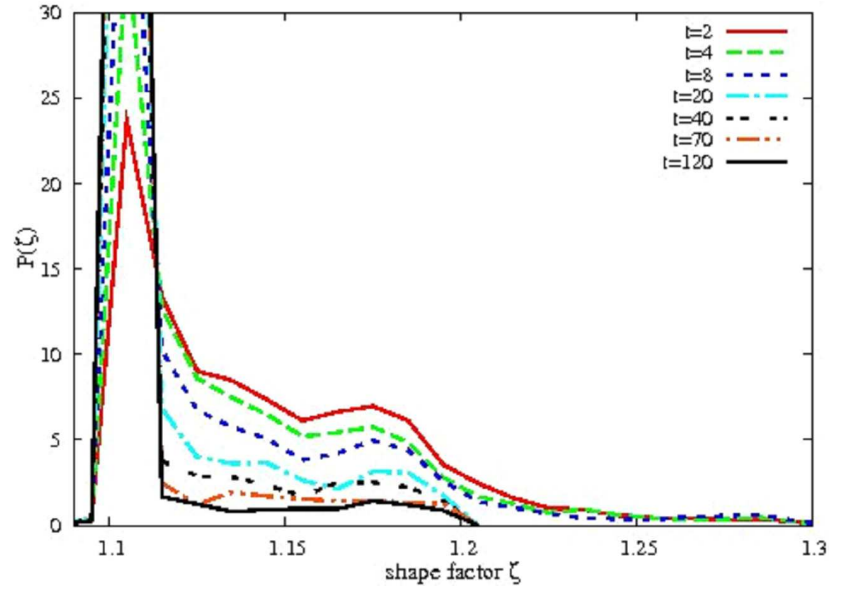

Fig. 5. The temporal evolution of the probability distribution $P(\zeta)$ of the shape factor $\zeta$ for the less dissipative disks (B) at tapping intensity $\xi=0.7 \%$.

walls of the box, made of duraluminum, delimit a rectangular frame of height $H=340 \mathrm{~mm}$ and an adjustable width of typically $L=300 \mathrm{~mm}$. The box is secured on a heavy plane able to be inclined at different rates by means of a pulley system so that we could set an arbitrary inclination angle $\theta$ from the horizontal. The cylinders of diameter $d=4.00,5.00$, and $6.00 \pm 0.05 \mathrm{~mm}$ were used to prepare the monodisperse packings containing about 4500 grains.

The packing fraction $\rho$ is measured in a rectangular frame at distance $3 d$ from lateral walls of the box. Then $\rho$ is calculated considering the area contribution of each disk to this rectangular region. Disordered packings are prepared by pouring grains onto an initially horizontal glass plate at once. They are then spread with a knife edge until a flat layer is obtained, where the cylinders are randomly deposited without contact between them and at rest. The angle of the plane is then slowly increased up to an angle $\theta=90^{\circ}$, at constant angular velocity of $\approx$ $5^{\circ} \mathrm{s}^{-1}$. During the plane rotation, grains therefore freely slide downward and reach a mechanically stable state. The measured packing fractions of these disordered packings are $\rho=0.79 \div 0.80 \pm 0.01$. Partially ordered packings are obtained by using the same initial procedure followed by vibration of the inclined plane before starting the experiment with a hammer-like device installed below the container. The packing fraction of densely packed systems is $\rho=0.81 \div 0.86 \pm 0.01$. Those densities are far from the close packing limit $\rho_{c p}=\pi /(2 \sqrt{3}) \approx 0.91$. The packing fractions have been calculated from an average over 10 initial preparations of host packing.

The experimental study of collective rearrangements of grains requires to get a precise measurement of the grain positions. For this reason, the development of an accurate image processing technique has been a central aspect of the design of the experimental setup. During an experiment, the granular layer is repeatedly scanned 
by means of HP Scanjet 3800 . The scanner is firmly fixed to the plane narrowly below the bottom glass plate of the rectangular container. The scanner frame covers an area of $210 \times 297 \mathrm{~mm}$. The images are systematically acquired in resolution $600 \times 600$ dpi and 256 gray levels. Both center and diameter of each grain are accurately determined using the image processing program based on the Standard Hough Transform (SHT) [31]. In the output bitmap image, the diameters of grains are $d_{1} \approx 94,118$, and 142 pixels. This analysis allows one to detect both the centers and the diameters of cylinders with a high resolution of $0.04 \mathrm{~mm}$. More details about the experimental setup can be found in Ref. [32].

Let us now compare the simulation results of Figs. 2-5 to the experimental ones. Figure 6 shows the experimental result for the distribution $P(\zeta)$ of the shape factor $\zeta$, which corresponds to the packing of disks of diameter $d=6 \mathrm{~mm}$ at density $\rho=0.828$. Also included in Fig. 6 are the results from the numerical simulations for the less dissipative grains (B) after the second tap, at $\xi=0.7 \%$, and $3 \%$. The corresponding packing fractions are 0.825 and 0.830 , respectively. Here we see that the agreement between the simulation and the experimental results is very good. Similar degree of agreement has been found for all the experimentally studied values of density.

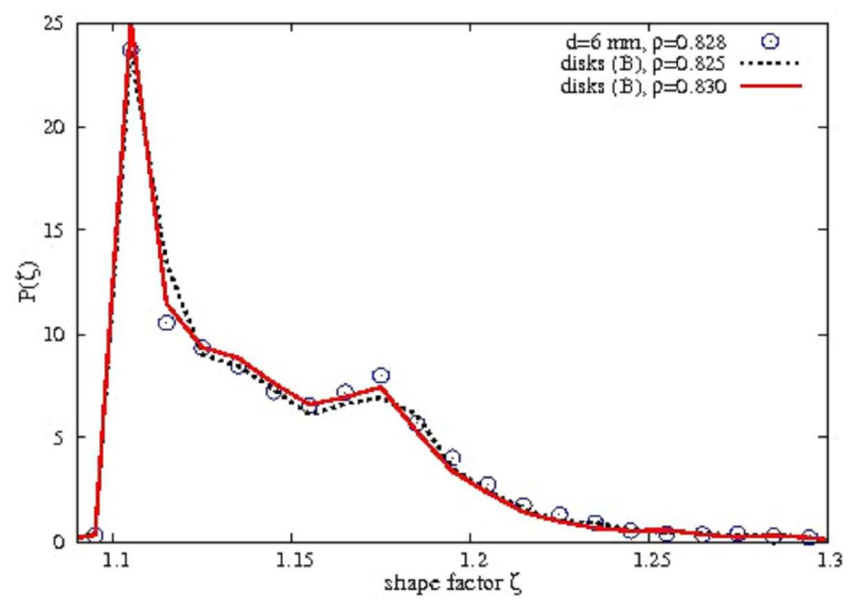

Fig. 6. Simulation (lines) and experimental (symbols) results for the probability distribution $P(\zeta)$ of the shape factor $\zeta$. Experimental result corresponds to the packing of disks of diameter $d=6 \mathrm{~mm}$ at density $\rho=0.828$. The simulation results correspond to the packings of less dissipative disks (B) at densities $0.825(\xi=0.7 \%)$ and $0.830(\xi=3 \%)$, after the second tap.

\section{Final remarks}

In this paper, we have reported some numerical and experimental results concerning the slow relaxation in granular media. Both the global evolution and the microscopic behaviour of the grains have been investigated. Our model is based on realistic granular dynamics. One of its main features is that during the second phase of the shake cycle the whole system is reassembled by using the event-driven moleculardynamics algorithm. We employed the Walton model $[13,14]$ that captures the major features of granular interactions. Our data show that the compaction dynamics strongly depends on the material properties of the grains. It was shown that the relaxation behavior is appreciably slowing down with the increase of the inelasticity of the grains.

We have shown that our simulations provide a direct microscopic observation of nonsequentially reorganized granular structures. The organization of grains at local level was studied by analyzing the time evolution of the probability distribution $P(\zeta)$ of the shape factor $\zeta$. It has been pointed out that this distribution is very sensitive to small structural changes of the system. The shape factor of Voronoi volumes has a distribution with a long tail that progressively reduces while the packing gets more compact. We have observed that the collective rearrangements of the grains in the two-dimensional system lead to growth of hexagonal domains [4].

We have also investigated a granular organization of two-dimensional packings at local level experimentally. The distributions $P(\zeta)$ of the shape-factor obtained numerically for the less dissipative grains (B), at various tapping intensities, are consistent with our experimental results.

\section{Acknowledgments}

This research was supported by the Ministry of Science of the Republic of Serbia, under Grants No. 141035 and No. 141003.

\section{References}

[1] J.B. Knight, C.G. Fandrich, C.N. Lau, H.M. Jaeger, S.R. Nagel, Phys. Rev. E 51, 3957 (1995).

[2] P. Philippe, D. Bideau, Europhys. Lett. 60, 677 (2002).

[3] P. Ribiere, P. Richard, D. Bideau, R. Delannay, Eur. Phys. J. E 16, 415 (2005).

[4] G. Lumay, N. Vandewalle, Phys. Rev. Lett. 95, 028002 (2005).

[5] G. Lumay, N. Vandewalle, Phys. Rev. E 74, 021301 (2006).

[6] G.C. Barker, A. Mehta, Phys. Rev. A 45, 3435 (1992).

[7] A. Mehta, G.C. Barker, J.M. Luck, J. Stat. Mech.: Theor. Exp. P10014 (2004).

[8] P. Philippe, D. Bideau, Phys. Rev. E 63, 051304 (2001).

[9] E. Kolb, J. Cviklinski, J. Lanuza, P. Claudin, E. Clement, Phys. Rev. E 69, 031306 (2004).

[10] F. Moučka, I. Nezbeda, Phys. Rev. Lett. 94, 040601 (2005).

[11] P.M. Reis, R.A. Ingale, M.D. Shattuck, Phys. Rev. Lett. 96, 258001 (2006).

[12] B.D. Lubachevsky, J. Comp. Phys. 94, 255 (1991). 
[13] O.R. Walton, R.L. Braun, J. Rheology 30, 949 (1986).

[14] O. Herbst, M. Huthmann, A. Zippelius, Granular Matter 2, 211 (2000).

[15] D. Goldman, M.D. Shattuck, C. Bizon, W.D. McCormick, J.B. Swift, H.L. Swinney, Phys. Rev. E 57, 4831 (1998).

[16] E. Falcon, C. Laroche, S. Fauve, C. Coste, Eur. Phys. J. B 3, 45 (1998).

[17] S. McNamara, E. Falcon, Phys. Rev. E 71, 031302 (2005).

[18] S.F. Foerster, M.Y. Louge, H. Chang, K. Allia, Phys. Fluids 6, 1108 (1994).

[19] J. Brujić, P. Wang, C. Song, D.L. Johnson, O. Sindt, H.A. Makse, Phys. Rev. Lett. 95, 128001 (2005).

[20] J.J. Brey, A. Prados, J. Phys.: Condens. Matter 13, 1 (2001)

[21] P. Richard, M. Nicodemi, R. Delannay, P. Ribiere, D. Bideau, Nat. Mater. 4, 121 (2005).

[22] D. Arsenović, S.B. Vrhovac, Z.M. Jakšić, Lj. Budinski-Petković, A. Belić, Phys. Rev. E 74, 061302 (2006).

[23] R. Hilfer, J. Non-Cryst. Solids 305, 122 (2002).
[24] G.C. Barker, A. Mehta, Phys. Rev. E 47, 184 (1993).

[25] W.L. Vargas, J.J. McCarthy, Phys. Rev. E 76, 041301 (2007).

[26] F. Aurenhammer, ACM Computing Surveys 23, 345 (1991)

[27] D.P. Fraser, M.J. Zuckermann, O.G. Mouritsen, Phys. Rev. A 42, 3186 (1990).

[28] A. Huerta, G.G. Naumis, Phys. Rev. Lett. 90, 145701 (2003).

[29] P. Richard, L. Oger, J.P. Troadec, A. Gervois, Phys. Rev. E 60, 4551 (1999).

[30] P. Richard, J.P. Troadec, L. Oger, A. Gervois, Phys. Rev. E 63, 062401 (2001)

[31] Brendan Drew, Hough Transform, From MathWorld — A Wolfram Web Resource, created by Eric W. Weisstein. http://mathworld.wolfram.com/HoughTransform.html

[32] Z.M. Jakšić, S.B. Vrhovac, B.M. Panić, Z. Nikolić, B.M. Jelenković, Eur. Phys. J. E 27, 345 (2008). 